\title{
Heart-shaped bubbles rising in anisotropic liquids
}

\author{
Chunfeng Zhou \\ Department of Chemical and Biological Engineering, University of British Columbia, Vancouver, \\ British Columbia V6T 1Z3, Canada \\ Pengtao Yue and James J. Feng ${ }^{\text {a) }}$ \\ Department of Chemical and Biological Engineering, University of British Columbia, Vancouver, \\ British Columbia V6T 1Z3, Canada and Department of Mathematics, University of British Columbia, \\ Vancouver, British Columbia V6T 1Z2, Canada \\ Chun Liu \\ Department of Mathematics, The Pennsylvania State University, University Park, Pennsylvania 16802 \\ Jie Shen \\ Department of Mathematics, Purdue University, West Lafayette, Indiana 47907
}

(Received 1 January 2007; accepted 9 March 2007; published online 27 April 2007)

\begin{abstract}
This Letter reports on numerical simulations motivated by experimental observations of an unusual inverted-heart shape for bubbles rising in an anisotropic micellar solution. We explain the bubble shape by assuming that the micelles are aligned into a nematic phase, whose anchoring energy on the bubble competes with the interfacial tension and the bulk elasticity of the nematic to modify the interfacial curvature. Numerical results show that bubbles with sufficiently strong planar anchoring rising in a vertically aligned nematic indeed assume the observed shape. The parameter values required are compared with the experimental materials and conditions. (C) 2007 American Institute of Physics. [DOI: 10.1063/1.2722421]
\end{abstract}

To investigate the impact of solids on viscoelastic liquids, Akers and Belmonte ${ }^{1}$ dropped spheres of diameter $d$ $\sim 1 \mathrm{~cm}$ into an aqueous solution of the worm-like micellar system cetylpyridinium chloride $(\mathrm{CPCl})$ /sodium salicylate (NaSal). Occasionally air bubbles were entrained into the fluid, and would rise in the wake of the ball (Fig. 1). Such a bubble assumes a peculiar shape while in the near wake, resembling an inverted heart or a spade (a). The upper surface has sloped shoulders that join in a point. The bottom is relatively flat with a small conical protrusion in the middle. As it rises, both points on top and bottom quickly retract and the bubble appears roughly spherical (b). Further up, the bubble assumes the familiar shape with a round top and a long pointed tail at the bottom (c). The last image resembles that seen of bubbles in viscoelastic polymer solutions, ${ }^{2,3}$ the tail being produced by the tensile stress in the wake of the bubble. The inverted-heart shape in the first image, on the other hand, has never been reported before. A possible explanation is that the micellar solution has been temporarily transformed into an anisotropic nematic liquid in the near wake of the falling ball. The ordered micelles have a preferred orientation, known as the "easy direction," with respect to the bubble surface, deviation from which is penalized by an anchoring energy. 5 " Such surface anchoring may compete with the interfacial tension and the bulk molecular order and force the bubble into the peculiar shape. Farther away from the ball, the micelles relax and lose the nematic order, and the bubble shape reverts to that commonly seen in viscoelastic liquids.

\footnotetext{
${ }^{a}$ Author to whom correspondence should be addressed. Electronic mail: jfeng@CHML.UBC.CA
}

Although Akers and Belmonte did not present direct evidence for the orientational order in the near wake, a flowinduced nematic state can be inferred from two facts. First, a falling ball produces strong elongation in its wake that tends to modify the microstructural conformation of the fluid. Both flexible polymers and worm-like micelles have been observed to align into "birefringent strands" in the near wake., Second, semidilute and concentrated micellar solutions are known to undergo an isotropic-to-nematic transition under shear, for surfactant concentrations down to $1.09 \mathrm{wt} . \% .^{9-11}$ At higher concentrations, micelles commonly exhibit a nematic phase even in equilibrium. ${ }^{12,13}$ The $\mathrm{CPCl}$ concentration used by Akers and Belmonte is estimated at $2.87 \mathrm{wt} . \%$, well into the semidilute regime. It is therefore reasonable to assume an anisotropic nematic state in the near wake, with the micelles predominantly oriented vertically. In such an environment, the bubble shape is affected not only by the hydrodynamic forces and interfacial tension, but also by the surface anchoring and bulk molecular orientation.

To test this hypothesis, we have carried out dynamic simulations of bubbles rising in a nematic fluid having a vertical far-field orientation. The nematic background is assumed to be time-invariant since the time required for the bubble to traverse the nematic region, estimated from its rise velocity, is much longer than the time scale of the simulation (cf. Fig. 3 below). The rheology and orientation of the fluid are modeled by the Leslie-Ericksen theory for liquid crystals, ${ }^{4}$ and the moving and deforming bubble surface is captured in a diffuse-interface framework. ${ }^{14}$ Details about the theoretical model and numerical method can be found elsewhere, ${ }^{15,16}$ and only a brief summary is given here. The free energy of a Newtonian-nematic mixture has three con- 

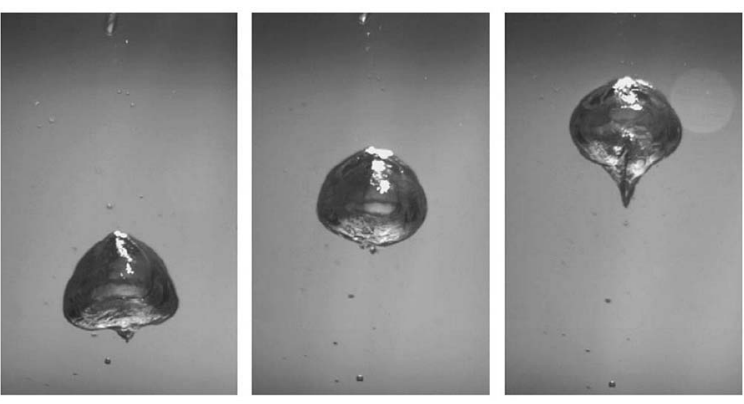

FIG. 1. An air bubble rising in the wake of a falling steel ball in a micellar solution. The bubble volume is roughly $2 \mathrm{~cm}^{3}$ and the images are separated by $33 \mathrm{~ms}$. From Akers and Belmonte (Ref. 1) with permission, (C2006 Elsevier B.V.

tributions: a mixing energy, a bulk elastic energy, and a surface anchoring energy

$$
\begin{aligned}
& f_{\text {mix }}=\frac{\lambda}{2}|\nabla \phi|^{2}+\frac{\lambda}{4 \epsilon^{2}}\left(\phi^{2}-1\right)^{2}, \\
& f_{\text {bulk }}=K\left[\frac{1}{2} \nabla \boldsymbol{n}:(\nabla \boldsymbol{n})^{T}+\frac{\left(|\boldsymbol{n}|^{2}-1\right)^{2}}{4 \delta^{2}}\right], \\
& f_{\text {anch }}=\frac{A}{2}(\boldsymbol{n} \cdot \nabla \phi)^{2} .
\end{aligned}
$$

In $f_{\text {mix }}, \phi$ is the phase-field variable with $\phi=1$ and -1 in the nematic and Newtonian bulk phases and $\phi=0$ at the interface, $\lambda$ is the mixing energy density and $\epsilon$ is the capillary width. $f_{\text {bulk }}$ is the Frank energy with a single elastic constant $K, \boldsymbol{n}$ being the director, regularized to permit defects where $|\boldsymbol{n}|$ deviates from unity over a small region of size $\delta .{ }^{4,17}$ For $f_{\text {anch, }}$, we adapt the Rapini-Popoular form ${ }^{5}$ for planar anchoring to our diffuse-interface formalism, with $A$ being the anchoring energy density and the easy direction being perpendicular to the interface normal $\nabla \phi$. Planar anchoring is expected for the micelles based on an entropic argument, ${ }^{12}$ which will be revisited when comparing the simulation with observations. In the sharp-interface limit, $2 \sqrt{2} \lambda / 3 \epsilon$ gives the interfacial tension $\sigma$ and $2 \sqrt{2} A / 3 \epsilon$ becomes the anchoring strength $W^{5,14,18}$

A variational procedure on the free energy, supplemented by the appropriate dissipative terms, leads to the governing equations: ${ }^{14}$

$$
\begin{aligned}
& \nabla \cdot \boldsymbol{v}=0, \\
& \rho\left(\frac{\partial \boldsymbol{v}}{\partial t}+\boldsymbol{v} \cdot \nabla \boldsymbol{v}\right)=-\nabla p+\nabla \cdot \boldsymbol{\sigma}, \\
& \frac{\partial \phi}{\partial t}+\boldsymbol{v} \cdot \nabla \phi=\gamma \lambda \nabla^{2}\left[-\nabla^{2} \phi+\frac{\phi\left(\phi^{2}-1\right)}{\epsilon^{2}}\right], \\
& \boldsymbol{h}=\gamma_{1} \boldsymbol{N}+\gamma_{2} \boldsymbol{D} \cdot \boldsymbol{n},
\end{aligned}
$$

where the deviatoric stress tensor

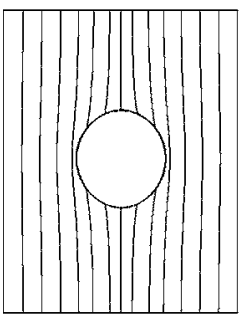

(a)

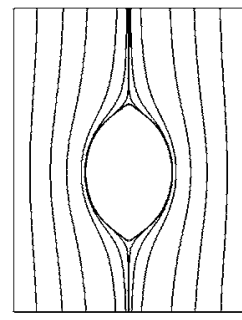

(b)

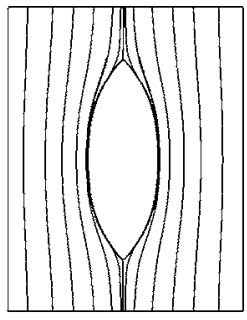

(c)
FIG. 2. Equilibrium shapes of stationary bubbles in a nematic with a vertical far-field orientation. The curves depict the director field and the bubbles are axisymmetric with planar anchoring. Both $\sigma$ and $K$ are fixed at $\sigma a / K=3$, and the three plots correspond to increasing anchoring strength: $\mathrm{Wa} / \mathrm{K}$ $=0.6,15$, and 150 .

$$
\begin{aligned}
\boldsymbol{\sigma}= & -\lambda(\nabla \phi \nabla \phi)-K \frac{1+\phi}{2}(\nabla \boldsymbol{n}) \cdot(\nabla \boldsymbol{n})^{T} \\
& -A(\boldsymbol{n} \cdot \nabla \phi) \boldsymbol{n} \nabla \phi+\frac{1+\phi}{2} \boldsymbol{\sigma}^{\prime}+(1-\phi) \mu \boldsymbol{D},
\end{aligned}
$$

with the Leslie viscous stress ${ }^{4} \quad \boldsymbol{\sigma}^{\prime}=\alpha_{1} \boldsymbol{D}: \boldsymbol{n n} \boldsymbol{n} \boldsymbol{n}+\alpha_{2} \boldsymbol{n} \boldsymbol{N}$ $+\alpha_{3} \boldsymbol{N} \boldsymbol{n}+\alpha_{4} \boldsymbol{D}+\alpha_{5} \boldsymbol{n} \boldsymbol{n} \cdot \boldsymbol{D}+\alpha_{6} \boldsymbol{D} \cdot \boldsymbol{n n}$, and the molecular field

$$
\begin{aligned}
\boldsymbol{h}= & K\left[\nabla \cdot\left(\frac{1+\phi}{2} \nabla \boldsymbol{n}\right)-\frac{1+\phi}{2} \frac{\left(|\boldsymbol{n}|^{2}-1\right) \boldsymbol{n}}{\delta^{2}}\right] \\
& -A(\boldsymbol{n} \cdot \nabla \phi) \nabla \phi .
\end{aligned}
$$

In the above, $\gamma$ is the Cahn-Hilliard mobility parameter, $\boldsymbol{D}$ $=\frac{1}{2}\left[\nabla \boldsymbol{v}+(\nabla \boldsymbol{v})^{T}\right]$ and $\boldsymbol{N}=d \boldsymbol{n} / d t-\frac{1}{2}\left[(\nabla \boldsymbol{v})^{T}-\nabla \boldsymbol{v}\right] \cdot \boldsymbol{n}$. The $\alpha$ 's are the Leslie viscous coefficients, $\gamma_{1}=\alpha_{3}-\alpha_{2}, \gamma_{2}=\alpha_{2}+\alpha_{3}$ $=\alpha_{6}-\alpha_{5}$, and $\mu$ is the viscosity of the Newtonian phase.

The governing equations are discretized on a finiteelement grid using the Petrov-Galerkin formulation with streamline upwinding for the constitutive equation. ${ }^{16}$ With axisymmetry, the 2D computational domain is covered by an unstructured grid of triangular elements. A key element of the numerical algorithm is an adaptive meshing scheme that deploys the finest grids around the interface and adaptively coarsens and refines the grid as the interface moves. Numerical experiments with grid refinement and time-step refinement have been carried out to ensure adequate resolution, and the accuracy and robustness of the code has been established by benchmarking against rising bubbles in Newtonian fluids and other published results. ${ }^{16}$

To analyze the effects of flow and molecular order separately, we first computed the equilibrium shape of a stationary bubble in a nematic (Fig. 2). The static shape depends on the competition among the interfacial tension $\sigma$, the anchoring strength $W$ and the bulk elastic energy $K$. Minimizing the total free energy, the bubble typically takes on a lemon shape [Fig. 2(b)], and the degree of elongation is determined by two dimensionless groups: $W / \sigma$ and $W a / K, a$ being the equivalent radius of the bubble. For weak anchoring $(\mathrm{Wa} / \mathrm{K}<1), \boldsymbol{n}$ readily deviates from the easy direction and bulk elasticity can exert little influence on the bubble shape [Fig. 2(a)]. For strong anchoring, however, the bubble becomes more elongated to reduce the bulk distortion at the expense of increased interface area [Fig. 2(c)]. Note the 


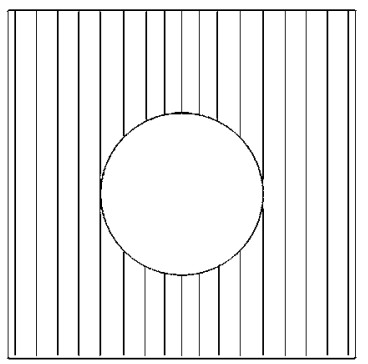

(a) $t=0$

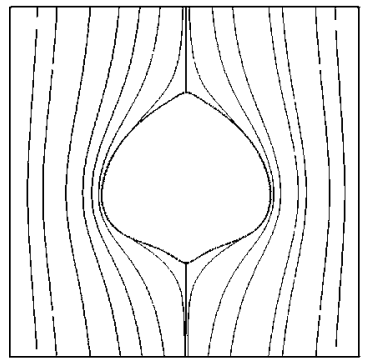

(c) $t=2.64$

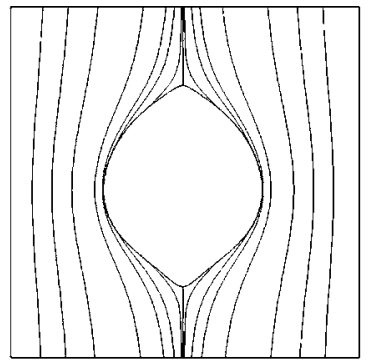

(b) $t=1.40$

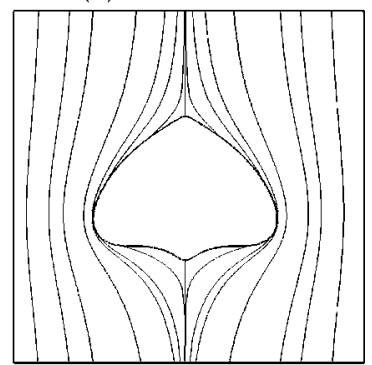

(d) $t=5.27$
FIG. 3. Snapshots of the rising bubble in a nematic with planar anchoring. The far-field molecular orientation is vertical. Eo=11.2, Mo=1.01, $W / \sigma$ $=5$, and $W a / K=15$. Time is scaled by $\sqrt{2 a / g}$. Steady state is reached in frame (d).

"boojum" defects at the poles of the bubble, where bulk distortion creates large surface curvatures. Both the lemon shape and the boojum defects have been reported in prior studies. $^{19,20}$

The shape of a rising bubble is also influenced by flow effects, expressed by two dimensionless groups: ${ }^{21}$ the Morton number $\mathrm{Mo}=g \eta^{4} / \rho \sigma^{3}$ and the Eötvos number Eo $=\rho g a^{2} / \sigma, \rho$ and $\eta$ being the liquid density and viscosity and $g$ the gravitational acceleration. In Akers and Belmonte's experiment, ${ }^{1}$ the micellar solution is strongly shear-thinning, and its surface tension $\sigma$ also varies depending on the relaxation and redistribution of the surfactants. Based on the data given, the experimental conditions correspond to $8.70<$ Eo $<20.3$ and $10^{-2}<\mathrm{Mo}<10^{3}$. The simulations will use Eo and Mo in these ranges, with the characteristic viscosity $\eta=\left(\alpha_{4}\right.$ $\left.-\alpha_{2}+\alpha_{6}\right) / 2$ being the average between two Miesowicz viscosities and the ratios of the Leslie coefficients approximating those of MBBA. ${ }^{4}$ The anchoring strength $W$ and bulk energy $K$ are more difficult to estimate. We have tested a range of $W / \sigma$ and $W a / K$ values, and their relevance to real materials will be discussed later. For computational conveniences, we have assigned equal density and viscosity to the "bubble" and matrix fluid; ${ }^{16}$ the buoyancy force is replaced by an upward body force acting on the bubble. The density and viscosity of the bubble phase are known to have only minor effects on the bubble shape. ${ }^{21}$

Figure 3 shows snapshots of a bubble during its rise. The initial shape is spherical, with a uniform director field [Fig. $3(\mathrm{a})]$. Within a time scale of $\eta a / W(=1.41$ in this case $)$, the director relaxes toward the easy direction on the bubble surface, deforming it into a lemon shape resembling Fig. 2(b). At this point the bubble velocity is about $46 \%$ of its terminal value. As the rise velocity increases, so does the hydrodynamic drag due to viscous and inertial forces. As a result, the shoulders are pushed down and flattened, and the bubble loses fore-aft symmetry [Fig. 3(c)]. Eventually a steady state is reached in Fig. 3(d), with a bubble shape closely resembling the experimental picture in Fig. 1(a). The terminal velocity $U$ corresponds to a Reynolds number $\operatorname{Re}=\rho U a / \eta$ $=3.39$, while the experimental value in Akers and Belmonte ${ }^{1}$ is estimated as $1.72<\operatorname{Re}<3.90$. The steady-state Ericksen number $\mathrm{Er}=\eta U a / K=2.05$. The flat bottom is reminiscent of the bubble shape in Newtonian liquids ${ }^{21}$ at a comparable Re, except for the protrusion in the middle due to the boojum defect. The dimensional time for Fig. 3(d) is about $200 \mathrm{~ms}$, which is consistent with the estimated experimental time required for the heart shape to develop. This simulation offers strong support for our hypothesis that it is the nematic order in the wake of the ball that affords the bubble its unusual shape.

Basic features of the inverted-heart shape-sloped shoulders and a flat bottom with a conical protrusion-are obtained if both $W$ and $K$ are sufficiently large; a rough guideline is $W \gtrsim \sigma$ and $K \gtrsim 0.1 \sigma a$. These numerical parameters need to be related to the experiment. ${ }^{1}$ Since no in situ characterization was done on the micellar solution, we are limited to circumstantial evidence. For nematic worm-like micelles, anchoring arises from entropic effects such as excluded volume, and a scaling argument on $W$ predicts strong planar anchoring $(W a / K \gg 1) .{ }^{12}$ This has been confirmed experimentally through quadrupolar interactions among colloidal particles. ${ }^{12,13}$ Thus, it is reasonable to assume planar anchoring in our computations, and the condition $W a / K \gg 1$ is consistent with the numerical parameters in Fig. 3. However, the estimated $W$ is smaller than the surface tension of common thermotropic liquid crystals. ${ }^{22}$ In the experiment, ${ }^{1}$ the abundance of surfactants may have reduced $\sigma$ below $W$. For lyotropic nematics made of self-assembled molecular aggregates similar to worm-like micelles, $W / \sigma>1$ has been observed for domains in a biphasic system. ${ }^{20,23}$

The mechanism revealed by the heart-shaped bubble has potential applications in other complex fluids that feature nematic-isotropic interfaces, such as nematic emulsions ${ }^{24}$ and polymer-dispersed liquid crystals (PDLC). ${ }^{25}$ In selfassembly of colloids for making photonic crystals, ${ }^{26}$ a nematic matrix will afford better control of the spatial periodicity as well as the possibility of nonspherical voids with better performance and tunability. ${ }^{27,28}$ In manufacturing PDLC films, planar anchoring inside nematic drops tends to produce a bipolar shape similar to those in Fig. 2. The drop shape and orientation can be exploited to enhance the contrast and switching speed between the on- and off-states. ${ }^{29,30}$ Finally, the phase morphology of bicontinuous polymerliquid crystal networks" and "reversed mode PDLC" 32 depends on the coupling between surface anchoring, bulk elasticity and deformation, and the mechanism discussed in this Letter is expected to play a key role.

Acknowledgment is made to the Donors of The Petroleum Research Fund, administered by the American Chemical Society, for partial support of this research. J.J.F. was also supported by the NSERC, the Canada Research Chair program and the Canada Foundation for Innovation. C.Z. ac- 
knowledges partial support by a University Graduate Fellowship from UBC.

${ }^{1}$ B. Akers and A. Belmonte, "Impact dynamics of a solid sphere falling into a viscoelastic micellar fluid," J. Non-Newtonian Fluid Mech. 135, 97 (2006).

${ }^{2}$ Y. J. Liu, T. Y. Liao, and D. D. Joseph, "A two-dimensional cusp at the trailing edge of an air bubble rising in a viscoelastic liquid," J. Fluid Mech. 304, 321 (1995).

${ }^{3}$ J. R. Herrera-Velarde, R. Zenit, D. Chehata, and B. Mena, "The flow of non-Newtonian fluids around bubbles and its connection to the jump discontinuity," J. Non-Newtonian Fluid Mech. 111, 199 (2003).

${ }^{4}$ P. G. de Gennes and J. Prost, The Physics of Liquid Crystals (Oxford, New York, 1993).

${ }^{5}$ A. Rapini and M. Popoular, "Distortion d'une lamelle nematique sous champ magnetique conditions d'ancrage aux parois," J. Phys. (Paris), Colloq. 30, 54 (1969).

${ }^{6}$ A. D. Rey, "Viscoelastic theory for nematic interfaces," Phys. Rev. E 61, $1540(2000)$.

${ }^{7}$ O. G. Harlen, "High-deborah-number flow of a dilute polymer solution past a sphere falling along the axis of a cylindrical tube," J. NonNewtonian Fluid Mech. 37, 157 (1990).

${ }^{8}$ N. Z. Handzy and A. Belmonte, "Oscillatory rise of bubbles in wormlike micellar fluids with different microstructures," Phys. Rev. Lett. 92, 124501 (2004).

${ }^{9}$ J.-F. Berret, D. C. Roux, and G. Porte, "Isotropic-to-nematic transition in wormlike micelles under shear," J. Phys. II 4, 1261 (1994).

${ }^{10}$ I. A. Kadoma and J. W. van Egmond, "Flow-induced nematic string phase in semidilute wormlike micelle solutions," Phys. Rev. Lett. 80, 5679 (1998).

${ }^{11}$ P. Fischer, E. K. Wheeler, and G. G. Fuller, "Shear-banding structure orientated in the vorticity direction observed for equimolar micellar solution," Rheol. Acta 41, 35 (2002).

${ }^{12}$ P. Poulin, N. Francès, and O. Mondain-Monval, "Suspension of spherical particles in nematic solutions of disks and rods," Phys. Rev. E 59, 4384 (1999).

${ }^{13}$ O. Mondain-Monval, J. C. Dedieu, T. Gulik-Krzywicki, and P. Poulin, "Weak surface energy in nematic dispersions: Saturn ring defects and quadrupolar interactions," Eur. Phys. J. B 12, 167 (1999).

${ }^{14}$ P. Yue, J. J. Feng, C. Liu, and J. Shen, "A diffuse-interface method for simulating two-phase flows of complex fluids," J. Fluid Mech. 515, 293 (2004).

${ }^{15}$ P. Yue, J. J. Feng, C. Liu, and J. Shen, "Interfacial force and Marangoni flow on a nematic drop retracting in an isotropic fluid," J. Colloid Interface Sci. 290, 281 (2005).

${ }^{16}$ P. Yue, C. Zhou, J. J. Feng, C. F. Ollivier-Gooch, and H. H. Hu, "Phase- field simulations of interfacial dynamics in viscoelastic fluids using finite elements with adaptive meshing," J. Comput. Phys. 219, 47 (2006).

${ }^{17}$ C. Liu and N. J. Walkington, "Approximation of liquid crystal flows," SIAM (Soc. Ind. Appl. Math.) J. Numer. Anal. 37, 725 (2000).

${ }^{18} \mathrm{R}$. Yamamoto, "Simulating particle dispersions in nematic liquid-crystal solvents," Phys. Rev. Lett. 87, 075502 (2001).

${ }^{19}$ Q. Shen, C. Liu, and M. C. Calderer, "Axisymmetric configurations of bipolar liquid crystal droplets," Continuum Mech. Thermodyn. 14, 363 (2002).

${ }^{20}$ Y. A. Nastishin, H. Liu, T. Schneider, V. Nazarenko, R. Vasyuta, S. V. Shiyanovskii, and O. D. Lavrentovich, "Optical characterization of the nematic lyotropic chromonic liquid crystals: Light absorption, birefringence, and scalar order parameter," Phys. Rev. E 72, 041711 (2005).

${ }^{21}$ J. R. Grace, T. Wairegi, and T. H. Nguyen, "Shapes and velocities of single drops and bubbles moving freely through immiscible liquids," Trans. Inst. Chem. Eng. 54, 167 (1976).

${ }^{22}$ A. A. Sonin, The Surface Physics of Liquid Crystals, 1st ed. (Gordon and Breach, Amsterdam, 1995).

${ }^{23}$ A. V. Kaznacheev, M. M. Bogdanov, and A. S. Sonin, "The influence of anchoring energy on the prolate shape of tactoids in lyotropic inorganic liquid crystals," J. Exp. Theor. Phys. 97, 1159 (2003).

${ }^{24}$ T. Tixier, M. Heppenstall-Butler, and E. M. Terentjev, "Spontaneous size selection in cholesteric and nematic emulsions," Langmuir 22, 2365 (2006).

${ }^{25}$ M. Mucha, "Polymer as an important component of blends and composites with liquid crystals," Prog. Polym. Sci. 28, 837 (2003).

${ }^{26}$ V. N. Manoharan, A. Imhof, J. D. Thorne, and D. J. Pine, "Photonic crystals from emulsion templates," Adv. Mater. (Weinheim, Ger.) 13, 447 (2001).

${ }^{27}$ V. G. Nazarenko, A. B. Nych, and B. I. Lev, "Crystal structure in nematic emulsion," Phys. Rev. Lett. 87, 075504 (2001).

${ }^{28}$ K. P. Velikov, T. van Dillen, A. Polman, and A. van Blaaderen, "Photonic crystals of shape-anisotropic colloidal particles," Appl. Phys. Lett. 81, 838 (2002).

${ }^{29}$ P. S. Drzaic and A. Muller, "Droplet shape and reorientation fields in nematic droplet/polymer films," Liq. Cryst. 5, 1467 (1989).

${ }^{30}$ P. K. Chan, K.-W. D. Lee, and T. L. Tran, "The effects of elongated nematic bipolar droplet orientation on the performance of polymerdispersed liquid crystal films," Comput. Mater. Sci. 21, 329 (2001).

${ }^{31}$ H. K. Jeong, H. Kikuchi, and T. Kajiyama, "Molecular weight dependence of hysteresis in electro-optic switching of (polymer/liquid crystal) composite systems," Polym. J. (Tokyo, Jpn.) 31, 974 (1999).

${ }^{32}$ M. Macchione, D. Cupelli, G. de Filpo, F. P. Nicoletta, and G. Chidichimo, "Morphology and electrooptic properties of reverse mode polymer dispersed liquid crystals," Liq. Cryst. 27, 1337 (2000). 